Gut, 1988, 29, 1591-1594

\title{
Effect of stress on oesophageal motor function in normal subjects and in patients with the irritable bowel syndrome
}

\author{
E E SOFFER, P SCALABRINI, C E POPE II, AND D L WINGATE \\ From the Gastrointestinal Science Research Unit, The London Hospital Medical College, London
}

SUMMARY Stress can modulate the motor function of the stomach, small bowel, and colon in healthy subjects, and of the small bowel and colon in patients with the irritable bowel syndrome (IBS). The effect of stress on oesophageal motility in eight healthy subjects and in eight IBS patients was studied, using two pressure transducers positioned just above the lower oesophageal sphincter and $5 \mathrm{~cm}$ proximally. Stressors were: a video arcade game, delayed audio feedback, and hand immersion in cold water. Each stress period was followed by five swallows of water. Frequency and amplitude of oesophageal contractions and the number of simultaneous and multipeaked contractions were manually counted for each stress period and compared to the preceding rest period. Frequency of contractions (per minute) tended to decrease during stress periods, but achieved significance only with the video arcade game in the control group $(2.0(0.6) v 1.2(0.4) ; p<0.01)$. No other trend was evident in either control or IBS patients. No abnormalities of oesophageal body function were recorded in IBS patients either in basal conditions, or under stress. Unlike the more complex motor programmes elsewhere in the gut, the preprogrammed nature of oesophageal peristalsis is not modulated by stress.

There is a convincing body of evidence that stress can exert a significant effect on the motor function of the gut: and may be used to modulate gastric, ${ }^{12}$ small bowel,,$^{34}$ and colonic ${ }^{56}$ motor activity in healthy subjects. The effect of stress on oesophageal motility is less well documented. An early study has suggested that emotional stress increased the percentage of deglutition induced simultaneous contractions in some, but not all volunteer subjects. ${ }^{7}$ Psychological stress was recently found to increase the amplitude of oesophageal contractions. ${ }^{8}$

Stress has been shown to modulate small bowel and colonic motor activity in patients with IBS. ${ }^{69}$ These studies suggest that the effects of stress are even more marked in those patients than in normal subjects. Because stress appears to affect oesophageal motility in normal subjects, this could be even more pronounced in IBS patients. This study was carried out in order to determine the effect of

Address for correspondence: D L Wingate, DM, FRCP, Director, GI Science Research Unit, 26 Ashfield Street, London E1 2AJ.

Received for publication 19 May 1988. established stressors on oesophageal motility in normal subjects and in IBS patients.

\section{Methods}

\section{SUBJECTS}

The control group comprised eight healthy volunteers, four men, aged 19 to 30 years. None of them had a history of psychiatric illness, chronic gastrointestinal symptoms, or use of medications. Three subjects were smokers, but smoking was not allowed during the study. There were eight patients in the IBS group (five men, three women) aged 27 to 75 years. All had symptoms of abdominal pain and change in bowel habits, lasting for more than one year, for which investigations had found no pathological cause. There was no history of psychiatric illness in any member of this group. The use of anticholinergics and antispasmodics was stopped for 24 hours before the study. Study protocol was approved by the local ethical committee, and subjects gave informed written consent. 
STRESSORS

Three stressors were used in the study.

\section{Psychological stressors}

Asteroids de luxe is an electronic arcade video game and the subject, facing a visual display which depicts a rocket menaced by asteroids and hostile spacecraft, operates five buttons that control the movement of the spaceship and fires laser beams at the aggressors. The game, enhanced by loud sound effects, requires considerable dexterity and concentration; points are scored for the destruction of aggressors. With higher scores the game becomes more difficult, thereby maintaining the stressor effect.

Delayed auditory feedback involves fluency of speech which depends upon the continuous monitoring of sound through an intact hearing system; distortion of voice or hearing leads to abnormality of speech. When auditory feedback is delayed, speech is incorrectly perceived, and attempted compensation leads to distortion of speech. Delayed auditory feedback (DAF) makes reading aloud difficult and unpleasant. ${ }^{10}$ Using a reel-to-reel tape recorder with a third tape head (Teac 32B), a delay of up to $0.17 \mathrm{~s}$ can be imposed on an audio signal. Each subject had to read from a standard text into a microphone connected to the input of the recorder: the delayed output from the third head was fed back to the subject through stereo headphones.

As mental concentration, associated with the stressors, could have an effect on oesophageal motility independent of that of the stressor, the subjects were asked to perform simple mathematical functions (addition, multiplication, and division of large numbers) in a separate period.

\section{Pain stressor}

Hand immersion in cold water $\left(4^{\circ} \mathrm{C}\right)$ was repeated at one minute intervals, and the hand removed for 15 seconds between immersions. The test lasted for 10 minutes.

\section{OESOPHAGEAL MOTILITY}

Oesophageal motility was measured by two pressure sensitive transducers, spaced $5 \mathrm{~cm}$ apart, mounted on a thin, $2 \mathrm{~mm}$ od, catheter (Gaeltec Limited, Dunvegan, Isle of Skye, UK). The catheter was passed transnasally and the distal transducer was positioned just above the lower oesophageal sphincter. Pressure signals from the transducers were encoded in pulse mode and stored on magnetic tape using a miniature data cassette tape recorder (Medilog 4-24, Oxford Medical Systems, Abingdon, Oxon, UK), which the subject carried in a shoulder bag. The subjects were thus fully ambulatory throughout the study. At the end of each study the tape was run through a replay unit, and data decoded and transferred to a chart recorder.

STUDY PROTOCOL

Studies were conducted in the morning after an overnight fast. After a control period of 30 minutes the volunteers were subjected to the various stressors. Each stress period lasted approximately 30 minutes, and was followed by a rest period of equivalent duration. The sequence of events was randomised except that the cold water test was always left to the end, and lasted 10 minutes. Each event was followed by five swallows of water, of $10 \mathrm{ml}$ volume, with a time interval of 30-60 seconds between swallows.

\section{ANALYSIS OF DATA}

The graphic records were divided into segments covering periods of stress and control and could only be identified by a code; analysis was carried by an observer who was unaware of the conditions of the study relating to each segment. Frequency of contractions, amplitude of contractions (observed throughout the various periods), amplitude of wet swallows (in response to $10 \mathrm{ml}$ of water given at the end of each stressor), and number of simultaneous and multi-peaked (defined as having three of more peaks) contractions were manually counted for each period. After the code was broken, the data for each stress period were compared with the data of the control period immediately preceding it.

\section{STATISTICAL ANALYSIS}

The median value was used to express frequency and amplitude of dry swallows in the various periods, in each subject. The median values were compared by the paired Student's $t$ test to assess the significance of change for each stress variable, and 0.05 was chosen as the level of significance required for rejection of the null hypothesis. Results expressed as mean (1) SD.

\section{Results}

Over 5000 oesophageal contractions were analysed. The only significant effect elicited by any of the stressors was a consistent reduction in the frequency of contractions produced by the video arcade game in healthy controls but not in IBS patients (Figure). The other stressors had no significant effect on the frequency of contractions in either group (Table 1).

The amplitude of contractions in the various periods, and after wet swallows, was not significantly affected by the various stressors neither in control nor in the IBS group (Tables 1,2 ).

No substantial change in qualitative abnormalities 
Table 1 Frequency of contractions (per minute) and amplitude (in $\mathrm{mmHg}$ ) in the various periods

\begin{tabular}{lllllllll}
\hline Variable & Rest & Asteroids & Rest & DAF & Rest & Math & Rest & Water \\
\hline Frequency & & & & & & & & \\
Control & $2 \cdot 0+0 \cdot 6^{*}$ & $1 \cdot 2+0 \cdot 4$ & $1 \cdot 9+0 \cdot 7$ & $1 \cdot 6+0 \cdot 5$ & $1 \cdot 5+0 \cdot 9$ & $1 \cdot 5+1 \cdot 0$ & $1 \cdot 7+0 \cdot 8$ & $1 \cdot 5+0 \cdot 7$ \\
IBS & $1 \cdot 2+0 \cdot 5$ & $0 \cdot 9+0 \cdot 5$ & $1 \cdot 0+0 \cdot 2$ & $1 \cdot 2+0 \cdot 7$ & $1 \cdot 3+0 \cdot 4$ & $0 \cdot 9+0 \cdot 3$ & $1 \cdot 1+0 \cdot 5$ & $1 \cdot 3+0 \cdot 4$ \\
Amplitude & & & & & & & \\
Control & $39 \cdot 9+11 \cdot 3$ & $39 \cdot 0+17 \cdot 7$ & $35 \cdot 6+13 \cdot 6$ & $40 \cdot 3+16 \cdot 1$ & $34 \cdot 6+14 \cdot 6$ & $31 \cdot 8+12 \cdot 9$ & $36 \cdot 7+13 \cdot 8$ & $33 \cdot 7+10 \cdot 8$ \\
IBS & $25 \cdot 0+9 \cdot 5$ & $31 \cdot 3+13 \cdot 9$ & $27 \cdot 6+8 \cdot 9$ & $32 \cdot 9+13 \cdot 2$ & $27 \cdot 1+12 \cdot 4$ & $28 \cdot 4+9 \cdot 3$ & $28 \cdot 4+12 \cdot 2$ & $28 \cdot 0+10 \cdot 9$ \\
\hline
\end{tabular}

Each stress period is compared with the preceding rest period. ${ }^{*}=\mathrm{p}<0 \cdot 01 . \mathrm{DAF}=$ delayed auditory feedback. Math $=$ mathematics. Water $=$ cold water.

of contractions (simultaneous or multi-peaked) was observed under stress, with the largest increase in simultaneous contractions during stress being $26 \%$. No abnormalities of oesophageal body function were observed in IBS patients, either in basal conditions or under stress.

\section{Discussion}

In 1962 Rubin et al, using non-perfused catheters, studied oesophageal motility in five volunteers during neutral interviews, and during interviews in which material previously found to be upsetting to each of the volunteers was discussed. ${ }^{7}$ In three of the five subjects more simultaneous contractions were observed during the stress interviews. The manometric system in use at that time did not allow accurate estimation of peristaltic amplitude, duration, and could not detect multiple peaks. In a recent study by Richter $e t$
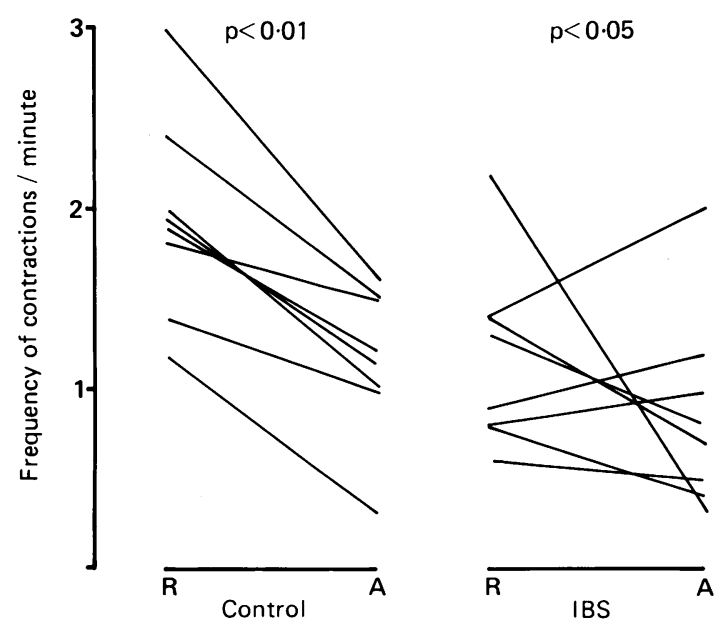

Figure Frequency of contractions in each subject, in both groups, during rest $(R)$ and the Asteroids de Luxe $(A)$ stress period following it. Each pair of data points is joined. The statistical significance $(P)$ of the difference between each set of paired data is given over the appropriate panel. $a l,{ }^{8}$ loud noise and difficult cognitive tasks used as stressors induced a modest $(10-17 \%)$ increase in the amplitude of oesophageal contractions during stress; they did not report any increase in simultaneous contractions previously reported during stress ${ }^{7}$ and after loud noise."

The results of our study suggest that mild stressors have little effect on oesophageal motility in normal subjects and IBS patients. This is in contrast with previous work showing that stress can affect the motility of other parts of the gut. Hand immersion in cold water was shown to delay the gastric emptying of a liquid meal in normal subjects. ${ }^{1}$ The mild psychological stressors (video arcade game and DAF) used in our study are known to induce a consistent reduction in the incidence of fasting migratory motor complexes in the small bowel of normal subjects, ${ }^{4}$ and may also evoke abnormal irregular small bowel contractile activity in IBS patients. ${ }^{9}$

We feel justified in comparing the results of our study with those mentioned above as we used the same stressors, and applied the same criteria for the selection of IBS patients, drawn from the same geographical area. We did not attempt to evaluate the extraintestinal effects of stress (cardiovascular responses, skin conductance, etc), or to measure subjective responses for several reasons. First, the measurements would result in repeated interruptions of each study. Second, there is no correlation between the magnitude of either the extraintestinal or subjective responses to stress and the magnitude of the gastrointestinal response. ${ }^{4}$ Finally, as mentioned

Table 2 Amplitude of contraction (in $\mathrm{mmHg}$ ) in response to wet swallows after the various stressors

\begin{tabular}{llllll}
\hline Group & Rest & Asteroids & DAF & Math & Cold water \\
\hline Control & $54 \cdot 1+26 \cdot 2$ & $54 \cdot 5+17 \cdot 1$ & $58 \cdot 4+22 \cdot 7$ & $42 \cdot 6+7 \cdot 5$ & $55 \cdot 9+25 \cdot 7$ \\
IBS & $40 \cdot 1+16 \cdot 3$ & $35 \cdot 7+9 \cdot 8$ & $38 \cdot 9+10 \cdot 2$ & $44 \cdot 8+13 \cdot 6$ & $48 \cdot 3+8 \cdot 6$
\end{tabular}

Each stress period is compared with the control rest period. No significant changes were observed under stress.

Math = mathematics. 
above, the same stressors have been used in our unit in the past and proved effective. ${ }^{44}$

The oesophagus is able to withstand another type of perturbation, that produced by the vestibular system. Motion sickness can be produced by placing a stationary subject in a rotatory drum. This produces tachygastria, as measured by surface electrodes, and if the stimulus is continued, nausea and vomiting ensues. ${ }^{12}$ Yet, perturbation of the vestibular system in control subjects during oesophageal manometry, using labyrinthine caloric stimulation, produced no qualitative difference in peristaltic activity as compared with the control period. ${ }^{13}$

One possible reason for the lack of response of the oesophagus to stress could be a different innervation of this organ compared with that of the stomach and the small bowel. Response of the gut to stress requires modulation of the activity of the enteric nervous system (ENS) by neural input from gut sensory receptors, and from the central nervous system through vagal efferents and spinal efferents through the preventebral ganglia. ${ }^{14}$ It is increasingly evident, however, that the ENS does not just provide the common effector pathway, but in the stomach and intestine, also has a complex information processing and programming function. ${ }^{15}$ The motor function of the oesophagus is in contrast confined to a single programme peristalsis which is reflex in character; in the oesophagus, the role of the ENS may be confined to the sequential contraction and relaxation of smooth muscle cells. It is of relevance hence, that a recent study of the myenteric plexus of the human gut has shown that the distal oesophagus, together with the proximal colon, are the least densely innervated regions in the gastrointestinal tract $;^{16}$ the opossum oesophagus has an equally sparse myenteric plexus. ${ }^{17}$ These findings are consistent with the lack of variation of motor activity in the oesophagus. One explanation for its apparent resistance to stress is that the selection of ENS programmes cannot be disrupted as there is only a single 'hard-wired' programme.

In conclusion, oesophageal contractile activity, in normal subjects and in IBS patients, was not affected by stressors previously shown to modulate gastric and small bowel motility. This would be due, in part, to the automatic and reflex nature of oesophageal motor activity.

\section{References}

1 Thompson DG, Richelson E, Malagelada J-R. Perturbation of upper gastrointestinal function by cold stress. Gut 1983; 24: 277-83.

2 Stanghellini V, Malagelada J-R, Zinsmeister AR, Go VLW, Kao PC. Stress-induced gastroduodenal motor disturbances in humans: possible humoral mechanisms. Gastroenterology 1983; 85: 83-91.

3 McRae S, Younger K. Thompson DG, Wingate DL. Sustained mental stress alters human jejunal motor activity. Gut 1982; 23: 404-9.

4 Valori RM, Kumar D, Wingate DL. Effects of different types of stress and of 'prokinetic' drugs on the control of the fasting motor complex in humans. Gastroenterology 1986; 90: 1890-900.

5 Almy TP. Experimental studies on irritable colon. Am J Med 1951; 10: 60-7.

6 Narducci F. Snape WJ, Battle WM, London RL, Cohen S. Increased colonic motility during exposure to a stressful situation. Dig Dis Sci 1985; 30: 40-4.

7 Rubin J, Nagler R, Spiro HM, Pilot LM. Measuring the effect of emotions on esophageal motility. Psychosom Med 1962; 24: 170-6.

8 Richter JE, Dalton CB, Katz PO, et al. Stress: a modulator of oesophageal contractions [Abstract]. Gastroenterology 1986; 90: A1603.

9 Kumar D, Wingate DL. The irritable bowel syndrome: a paroxysmal motor disorder. Lancet 1985; ii: 973-7.

10 Tacuber K, Appel E, Badian M. The delayed auditory feedback (DAF) method for inducing stress with betablockers and benzodiazepines. Psychopharmacol Bull 1980; 16: 74-5.

11 Stacher G, Steinringer H, Blau A, Landgraf M. Acoustically evoked esophageal contractions and defense reaction. Psychophysiology 1979; 16: 234-41.

12 Stern RM, Koch KL, Stewart WR, Lindblad IM. Spectral analysis of tachygastria recorded during motor sickness. Gastroenterology 1987; 92: 92-7.

13 Tibbling L, Hyden D. Vestibulo-vagal activity in the gastroesophageal region. In: Claussen CF, Kirtane MV, eds. Vertigo, nausea, tinnitus and hearing loss in cardiovascular diseases. Amsterdam: Elsevier Science Publishers, 1986: 201-5.

14 Wood JD. Enteric neurophysiology. Am J Physiol 1984; 247: G585-98.

15 Wingate DL. The brain-gut link. Viewpoints Dig Dis 1985; 17: 17-20.

16 Kumar D, Phillips SF. The human myenteric plexus. Gastroenterology 1987; 92: 1484.

17 Christensen J, Rick GA, Robison BA, Stiles MJ, Wix MA. Arrangement of the myenteric plexus throughout the gastrointestinal tract of the opossum. Gastroenterology 1983; 85: 890-9. 